\title{
Numerical investigation of geometrical corrugation influence to vortex flowfields at low Reynolds number
}

\author{
Yuya YAMAGUCHI*, Daisuke SASAKI*, Masato OKAMOTO*, \\ Koji SHIMOYAMA** and Shigeru OBAYASHI** \\ *Kanazawa Institute of Technology \\ 3-1 Yatsukaho, Hakusan, Ishikawa 924-0838, Japan \\ E-mail: b1406241@planet.kanazawa-it.ac.jp \\ **Institute of Fluid Science, Tohoku University \\ 2-1-1 Katahira, Sendai, Miyagi 980-8577, Japan
}

Received: 5 March 2019; Revised: 21 June 2019; Accepted: 18 November 2019

\begin{abstract}
Corrugated wings, which are cross-sectional shapes of dragonfly wing, are expected to improve aerodynamic performance due to vortices generated by irregular shapes at low Reynolds number region. It is difficult to observe the influence of vortices generated from the unevenness of the shape by experiments. In this research, the flowfields around corrugated wings were calculated using Cartesian mesh-based Computational Fluid Dynamics (CFD), Building-Cube Method (BCM). The simulation grasped the detailed flowfield, which was difficult to be visually observed by the experiment. From the visualization result, it is found that the flow circulation velocity inside the concave and convex surface is not accelerated but slow. The result indicates that the improvement in aerodynamic performance is caused by the shape and position of unevenness.
\end{abstract}

Keywords : Corrugated wings, Low Reynolds number, Cartesian mesh, Computational fluid dynamics, Visualization, Dragonfly airfoil

\section{Introduction}

Micro Air Vehicle (MAV) are often utilized for the disaster spot, ecosystem observation and topography investigation these days. Because the full length of the body is centimeter scale, the Reynolds number of MAV becomes low $\left(10^{2}-10^{4}\right)$. A laminar separation bubble is formed in low Reynolds number unlike a high Reynolds number domain because the viscous term becomes dominant. Numerical and experimental analysis of high performance airfoils in low Reynolds number flow is proposed (M. Anyoji et al., 2014). In low Reynolds number flows, flat plate or thin wings have better aerodynamic performance compared to thick streamlined airfoils. However, there are still many problems with related to the aerodynamic performance. For example, batteries tend to be heavy and the thrust is not sufficient in low Reynolds number. These results would be useful in designing low Reynolds number aircraft such as micro-air vehicles. For example, an insect-sized MAV was proposed by (Wood et al., 2012) and (Kroo and Kunz., 2001).

Many studies about the wings of insects have been conducted. The flow regimes are basically low Reynolds number region. For example, the wing of an insect is very thin and film like containing joints, and is not generally a streamlined wing. It is said that the dragonfly has good aerodynamic coefficients among a lot of insects. M. Okamoto et al.(1996) showed an example of the wing shape of the dragonfly.
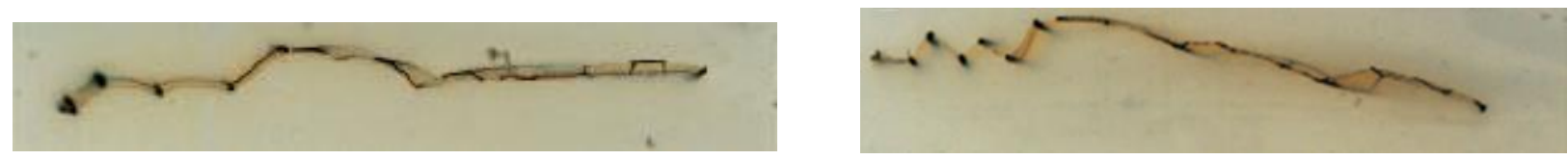

Fig.1 Example of dragonfly wing cross sections 
The wing of the dragonfly has an uneven shape as shown in Fig.1. The dragonfly produces vortices in the part of the uneven shape during a flight. It is estimated that the vortices make the aerodynamics performance better. However, the detailed principle and the factor of the good aerodynamic performance are unknown. Therefore, it is useful to investigate the influence of the uneven shape to flows and aerodynamic performance. In this study, a model created by (R. Fujii et al., 2017) is used, which was proposed for a structurally strong corrugated wings by (R. Fujii et al., 2017). A characteristic of this corrugated wing with related to aerodynamic performance is almost the same as a flat plate in the same extent low Reynolds number region, but it can be stiffer than a flat plate. Also, in order to investigate the influence of the vortex generated by the unevenness on the flowfield, two kinds of models with the closed uneven surface are newly prepared. We investigate the influence of the shape of concavo-convex on the flowfield using these two models. Therefore, fluid analysis of corrugated wings is carried out and the reason why high aerodynamic performance is achieved is investigated through the visualization.

\section{Method of calculation}

\subsection{Calculation conditions}

In this study, the computational value was compared with the experimental value at $\operatorname{Re}=10,000$. Here, the Reynolds number is based on a chord length of the corrugated model. The governing equation used in the present computation is incompressible Navier-Stokes equation and continuous equation. Although this study does not use the turbulence model and performs the Implicit LES with laminar flow, the flow also involves turbulent structures. This study employs Cartesian-mesh based solver, Building-Cube Method (BCM). The BCM method is proposed in K. Nakahashi et al., 2004. BCM divides a calculation domain into plural blocks called Cube, and Cells are generated in each Cube. The Cartesian cells are equally distributed in the Cube. The characteristics of BCM are a simple mesh generation for complicated shape geometries, parallel calculation, less numerical errors useful to capture small vortices. Because it is expected in this study that small vortices occur in an uneven shape part of the corrugated wing of the dragonfly.

Fractional step method is used for the present incompressible flow computation, where the space is calculated with third order accuracy, and the time is calculated with second order accuracy. Figure 2 shows flowchart of fractional step method. The boundary layer thickness is estimated by Eq. 1, which expects that the boundary layer thickness of the present computation is 0.00005 [m]. From this result, there are 16 mesh points assigned in the boundary layer. Therefore, it is possible to capture turbulence. Table 1 describes the mesh information used in this study. In the analysis, it is nondimensionalized with reference to the chord length (c). The analysis region is $48 \mathrm{c}$ in the vertical and horizontal directions and $1.5 \mathrm{c}$ in the span direction with respect to the chord length. The analysis condition is shown in Fig. 3. The boundary conditions for inflow and outflow are as summarized in Table 2. Table 2 corresponds to Fig.3. Also, periodic boundary conditions are applied as boundary conditions in the span direction. Three-dimensional fluid analysis of the corrugated wing is conducted in this study. Because the angles of attacks are set from 0.75 degree to 12 degree, three computational meshes are generated by considering the wake direction. Low Angle Of Attack (Hereinafter, the Angle Of Attack is expressed as AOA) mesh is suitable for the computation at the lower angles of attacks, where high resolution mesh is allocated around the geometry. As an example, the Cube allocation of low AOA mesh is shown in Fig. 4. In addition, the cell is evenly generated in each Cube where each Cube is divided by $16 \times 16 \times 16$. The details of the computation method can be refered to (D. Iioka et al., 2017).

$$
\delta=\frac{5 \times x}{\sqrt{R e}}
$$

$\delta$ : Thickness of boundary layer, Re: Reynolds number, $\mathrm{x}$ : Representative lengh 


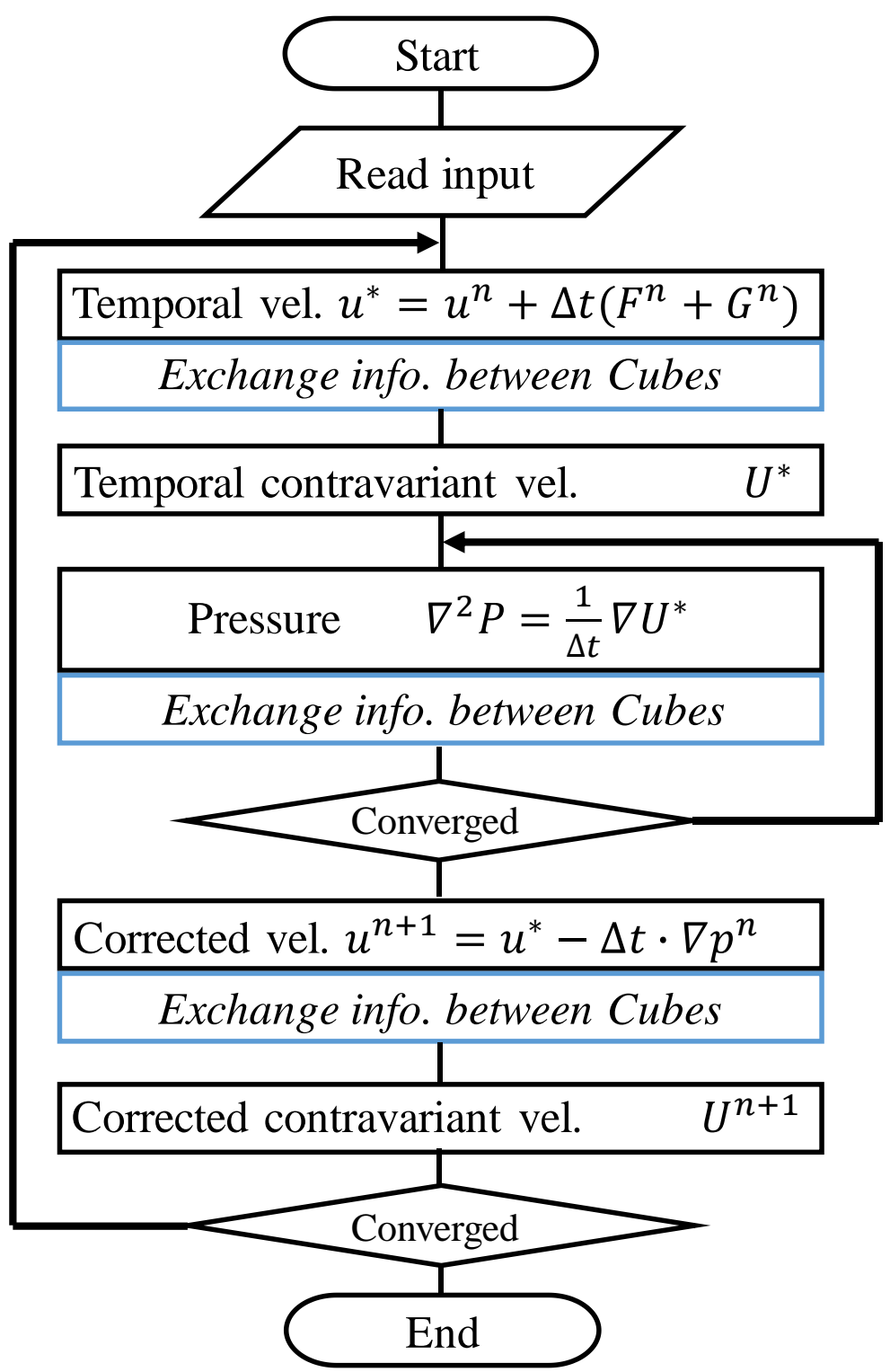

Fig.2 Flowchart of fractional step method

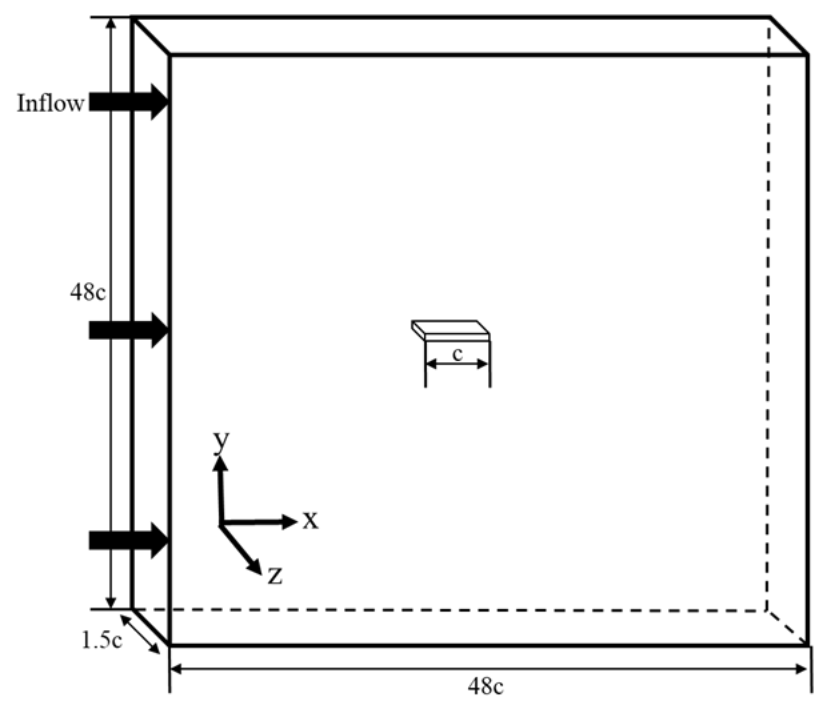

Fig.3 Computational domain 


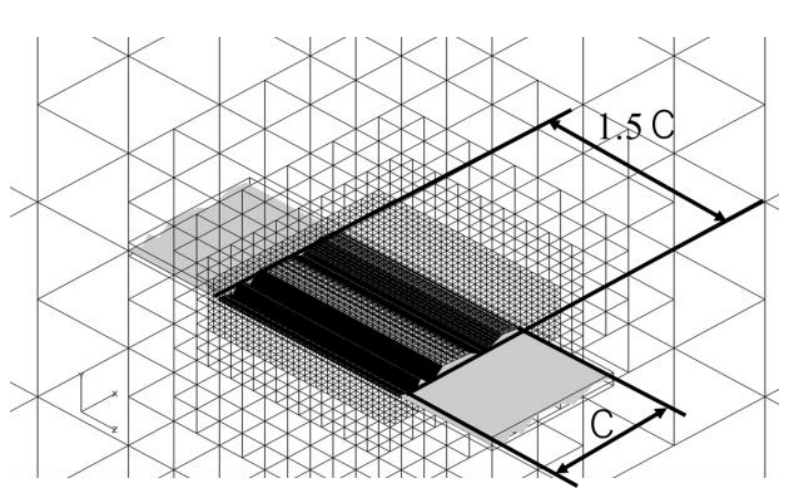

(a) Overview

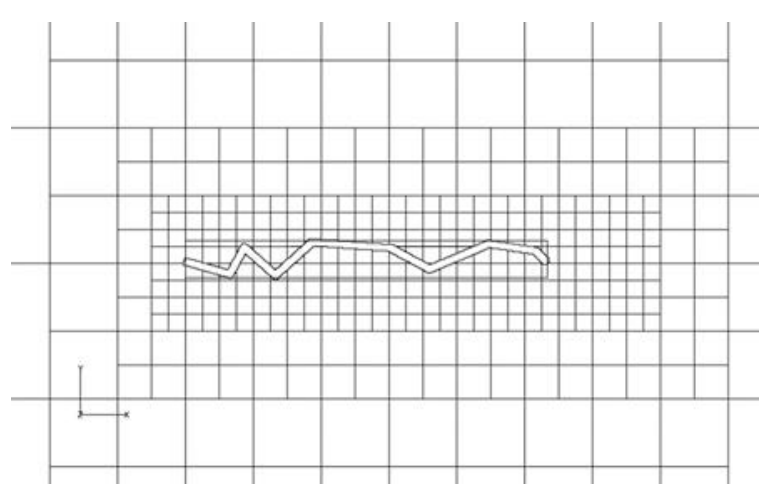

(b) Cross section view at $\mathrm{z}=0$

Fig.4 Cube allocation (low AOA mesh)

Table1 Mesh information

\begin{tabular}{c|c|c|c}
\hline Type of mesh & Negative AOA & Low AOA & High AOA \\
\hline $\begin{array}{c}\text { Number of Cubes } \\
\begin{array}{c}\text { Number of } \\
\text { cells per Cube }\end{array}\end{array}$ & 10,642 & 7,366 & 14,555 \\
\hline $\begin{array}{c}\text { Minimum cell } \\
\text { size }\end{array}$ & 0.002930 & 0.002930 & 0.002930 \\
\hline Outer size & $48 \times 48 \times 1.5$ & $48 \times 48 \times 1.5$ & $48 \times 48 \times 1.5$ \\
\hline
\end{tabular}

Table2 Boundary condition

\begin{tabular}{l|l|l}
\hline Axis & Velocity & Pressure \\
\hline$-X$ & Dirichlet & Neumann \\
\hline$+X$ & Convection outflow & Constant pressure \\
\hline$-Y$ & Neumann & Neumann \\
\hline$+Y$ & Neumann & Neumann \\
\hline$-Z$ & Periodic & Periodic \\
\hline$+Z$ & Periodic & Periodic \\
\hline
\end{tabular}

\subsection{Calculation model}

The calculation model is the thick corrugated wing in the article of Fujii (R. Fujii et al., 2017) shown in Fig. 5. The height of the original model is $0.09 \mathrm{c}$, thickness of the wings is $0.01 \mathrm{c}$. Chord length of the model in the experiment is $22[\mathrm{~mm}]$. In addition, the corrugated wings of the closed gap are also computed for comparison. The AOA in this model is based on the center line.

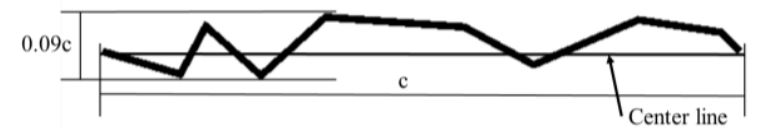

(a) Corrugated wing

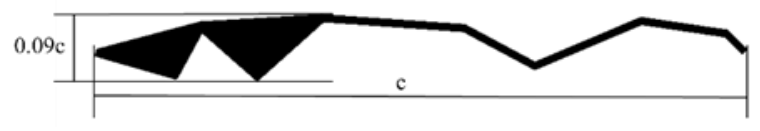

(b) Upper-closed corrugated wing

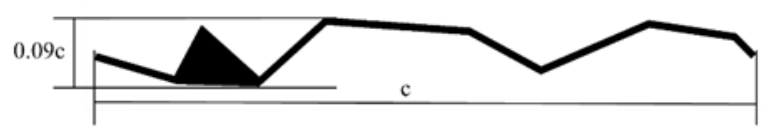

(c) Lower-closed corrugated wing

Fig. 5 Computational models 


\section{Calculated result of corrugated wing \\ 3.1 Comparison of aerodynamic coefficient}

From aerodynamic coefficients of the three-dimensional calculation shown in Fig. 6, lift coefficient and drag coefficient show a similar tendency to experimental value. The experimental values are quoted from (R. Fujii et al., 2017). The lift slope is less than the experimental value because it is difficult to match the zero lift angle for the tiny experimental model. The difference between the model geometry and the experiment are modelled using a stair-case representation of the present computation. In addition, the moment coefficient of analysis value about the $25 \%$ chord length from a leading edge is quite different from the experimental value at almost every AOA. From (a) of Fig. 6, the experimental values and the analysis values show close values at 0 degree and high AOA. At most angles of attack, the analysis value is smaller than the experimental value. This result indicates that separation is large in the experiment. Since the separation of experimental value is large, the center of pressure is rearward, and the head-down moment is large. This leads the moment coefficient of the experimental value negatively large. As the reason, the influence of shape and also the measurement accuracy of pitching moment by experiments are expected. However, the graph of lift coefficient and drag coefficient are very near to the experimental value. Therefore, it can be stated that the results of BCM used in this research is valid.

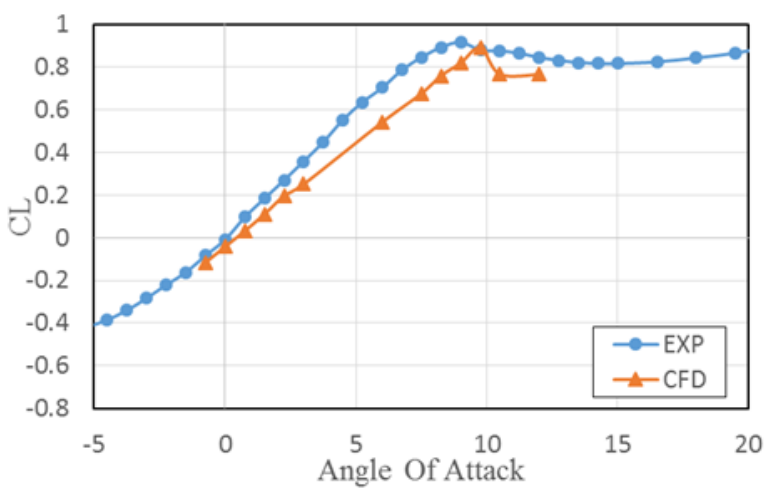

(a) Lift coefficient versus AOA

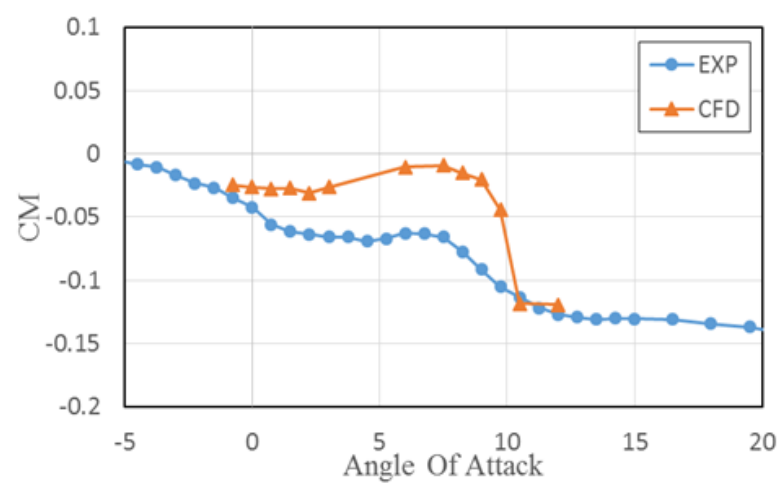

(c) Pitching moment coefficient versus AOA

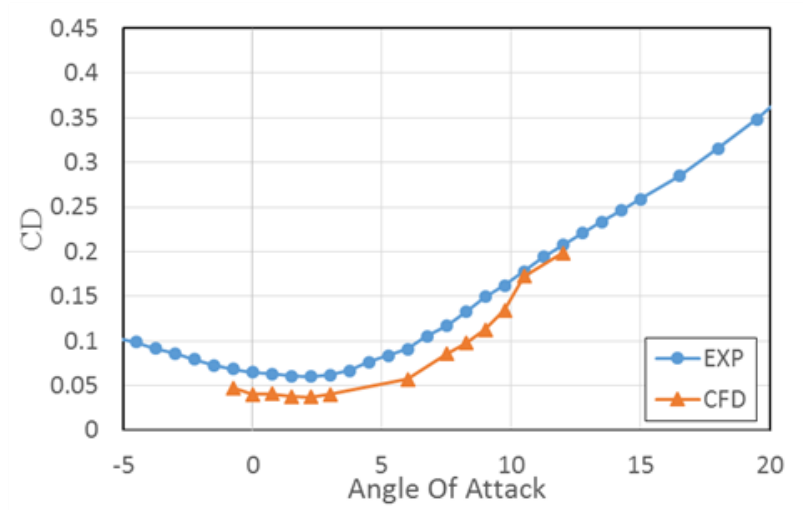

(b) Drag coefficient versus AOA

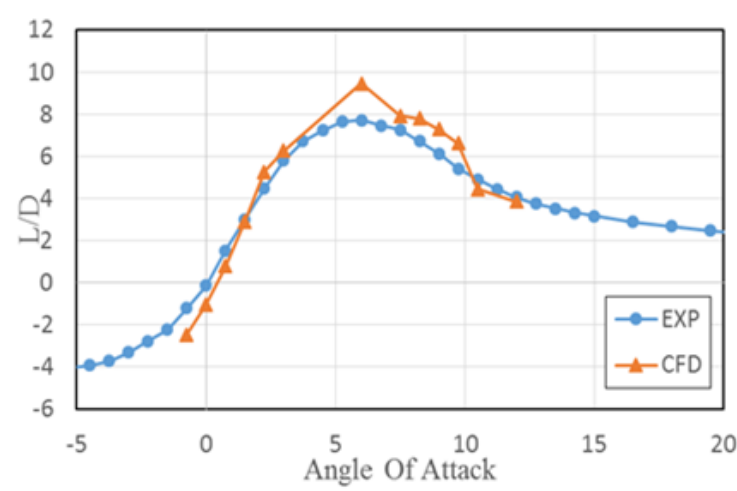

(d) L/D versus AOA

Fig.6 Aerodynamic coefficients

\subsection{Comparison of visualization}

A visualization diagram of the instantaneous value of the velocity obtained from the calculation result is shown in Fig. 7. In addition, all visualization figures are cross sections at the middle of the spanwise direction. From the lift coefficient graph in Fig. 6 (a), it can be seen that the stall AOA is 9.75[deg]. Therefore, velocity magnitude contours of the stall angle (9.75[deg]) and the higher angles after the stall (10.5[deg]) are compared in Fig. 7. At an AOA=9.75[deg], after the flow separates near the leading edge, it reattaches near the center of the chord. However, at the AOA=10.5[deg], the flow is separated near the leading edge and completely stalled.

Figure 8 shows the streamlines at the AOA of 3 and 9 [deg]. When the low AOA, the vortex flow is generated in the 
unevenness portion and it does not affect the entire flow much. It is concluded that the entire flowfield is two dimensional. On the other hand, at a high AOA, the vortices occurred in the irregular section with a three-dimensional form, and affects the entire flowfields. In addition, it was found that the vortices generated inside the irregularities of the corrugated wing were slow. Three-dimensional visualization is shown in Fig. 9. From this figure, it can be seen that a three-dimensional vortices are generated near the leading edge at a high AOA. This shows that the vortices occurred in the unevenness of corrugated becomes three-dimensional when the AOA increases.

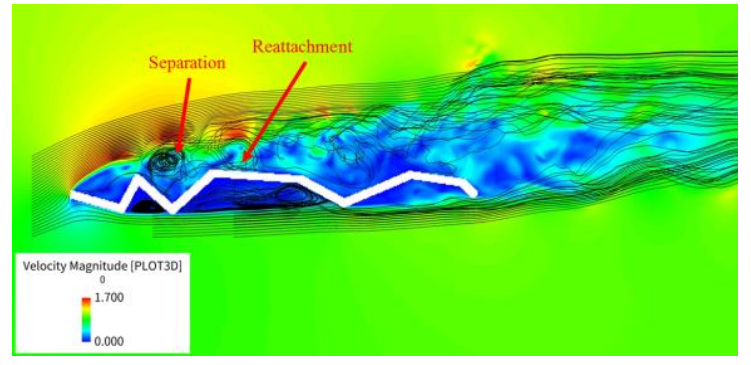

(a) $\mathrm{AOA}=9.75[\mathrm{deg}]$

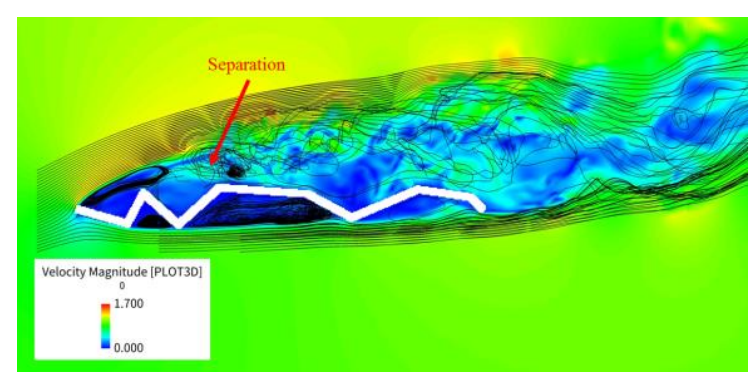

(b) $\mathrm{AOA}=10.5[\mathrm{deg}]$

Fig.7 Instantaneous velocity magnitude contours

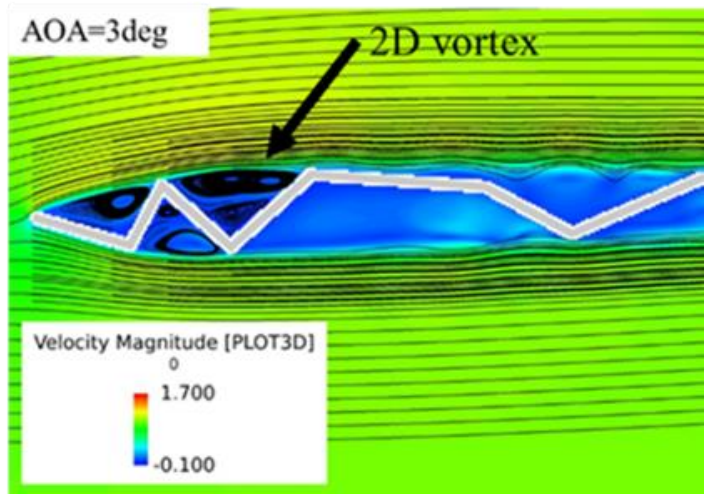

(a) $\mathrm{AOA}=3[\mathrm{deg}]$

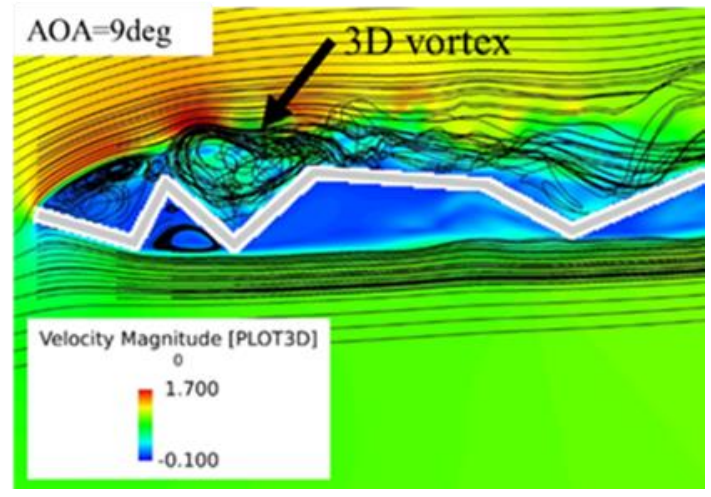

(b) $\mathrm{AOA}=9[\mathrm{deg}]$

Fig. 8 Instantaneous velocity magnitude contours with streamlines

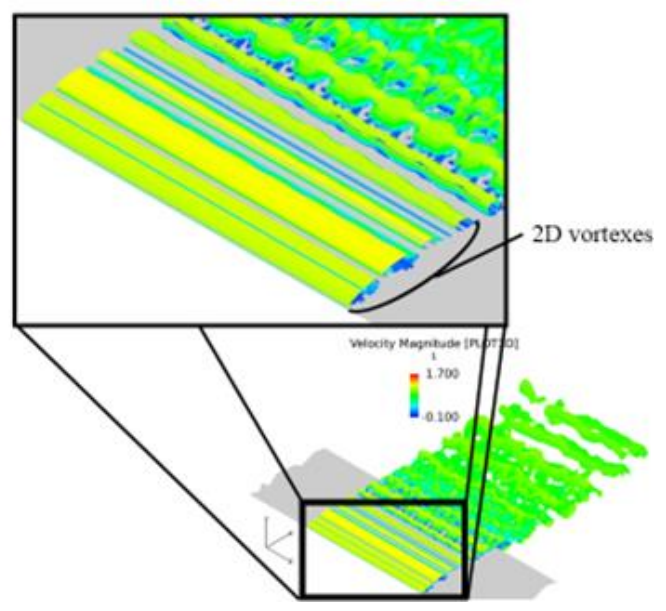

(a) $\mathrm{AOA}=3[\mathrm{deg}]$

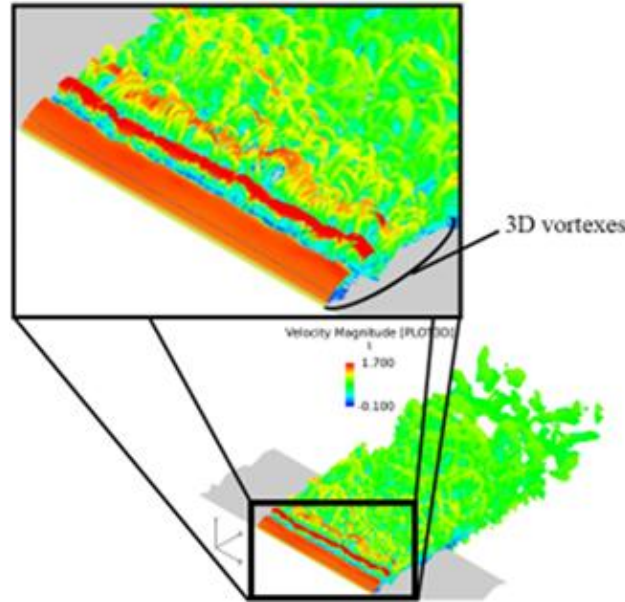

(b) $\mathrm{AOA}=9[\mathrm{deg}]$

Fig.9 Instantaneous velocity magnitude contours with three-dimensional view

\section{Effect of unevenness}

In order to investigate the influence of the unevenness of the corrugated wing to the improvement of the aerodynamic coefficient, two models shown in Fig. 5 are computed: upper-closed corrugated wing model and lower-closed corrugated 
wing model. The upper-closed corrugated wing is constructed by filling the upper gap, while the lower-closed one is constructed by filling the lower gap. The AOA of $6[\mathrm{deg}]$ is chosen for the comparison because the highest lift drag ratio is achieved at the angles, such as those in Fig. 6 (d).

The aerodynamic coefficients of those models are plotted in Fig. 10. Lift coefficient of upper-closed model is higher compared to the original model, while lower-closed model is close to the original. Drag coefficients of the three models are almost similar values. The flowfields are visualized in Fig. 11. It shows that the flowfields become more smooth by filling the upper gap, which suppress the generation of three-dimensional vortices. This leads the increase of lift for upper-closed model. On the other hand, the overall flowfields of lower-closed model are quite similar to the original model though the gap was filled. This is because the gap is relatively small and located close to the leading edge.

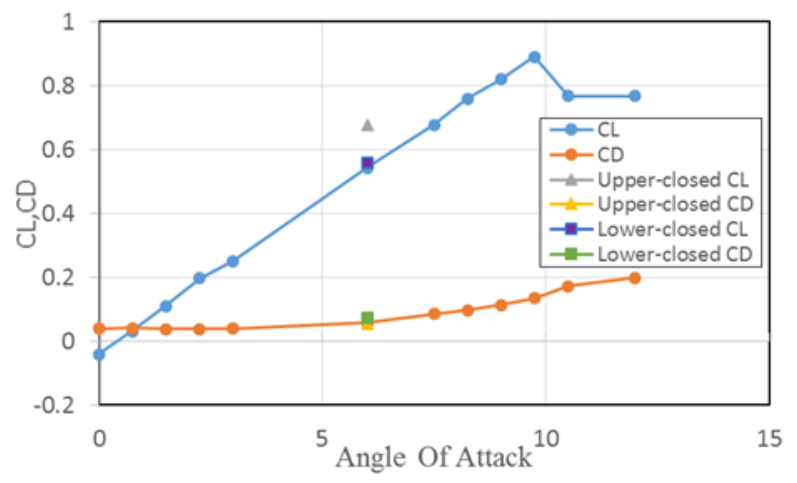

Fig.10 Aerodynamic coefficients versus AOA

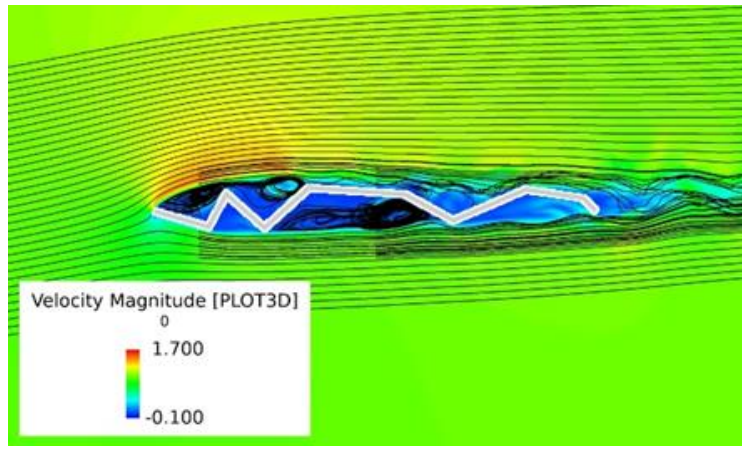

(a) Corrugated model

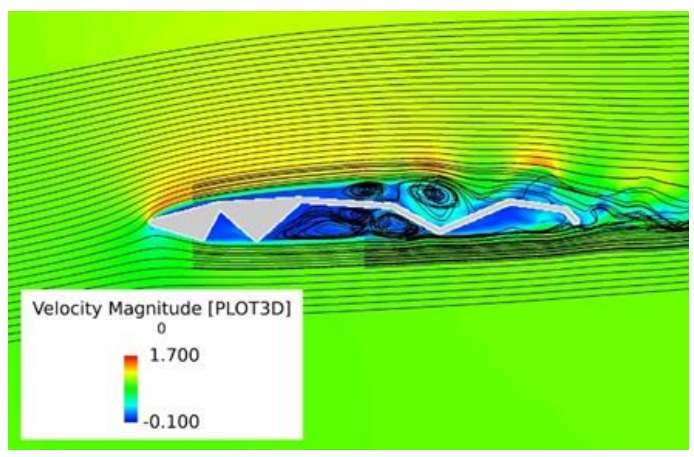

(b)Upper-closed model

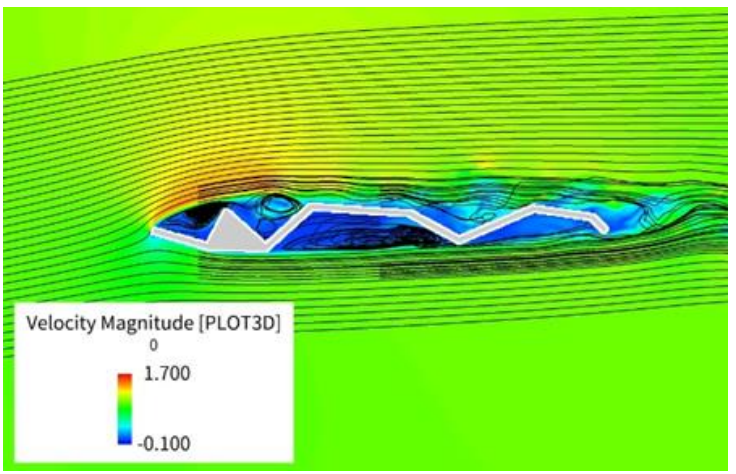

(c) Lower-closed model

Fig.11 Instantaneous velocity magnitude contours with streamline of three models

\section{Conclusion}

In this study, computational fluid analysis of corrugated wings was carried out. A comparison with experimental values was conducted to elucidate the vortices generated by the corrugation or unevenness of the shape. Comparison between 
the computational value and the experimental value shows that the computation can predict the tendency of aerodynamic coefficients well. From the visualization results, it is found that a two-dimensional vortex dominates the flowfields at low angles of attack, while a three-dimensional vortex is generated in the flowfields at high angles of attack. In addition, it is revealed that the existence of vortices inside the concavities and convexities or unevenness contributes to the improvement of corrugated wing. By computing the model with the closed upper concave part, the flowfields on the top surface are dominated by the two-dimensional vortex, and the aerodynamic coefficient is increased compared to the normal model. As a result, the lift coefficient improved in the model with the upper closed model. From this, it was found that the uneven shape of the upper surface of the corrugated wing is related to the improvement of the aerodynamic coefficient, especially the lift coefficient. It is newly found that the circulation speed or vortices inside the irregularities of the corrugated wing are slow. There were experimental studies in the past that have captured vortices occurred within the irregularities through visualization. This time it was able to capture the vortices inside the irregularity of corrugated wing through visualization with CFD.

\section{Acknowledgements}

This work was partly supported by JSPS KAKENHI Grant Numbers 17K05148 and 16K06894. Part of the work was carried out under the Collaborative Research Project of the Institute of Fluid Science, Tohoku University. The computations were carried out at the supercomputers of Advanced Fluid Information Research Centre, Institute of Fluid Science.

\section{References}

Anyoji, M., Nonomura, T., Aono, H., Oyama, A. and Fuji, K., Computational and experimental analysis of a highperformance airfoil under low-reynolds-number flow condition, Journal of Aircraft, Vol.51, No.6 (2014), pp.18641872.

Fujii, R. and Okamoto, M., Reynolds number effects of corrugated airfoil, 49th Fluid Dynamics Conference/35nd Aerospace Numerical Simulation Symposium, CD-ROM Proceedings of 49th Fluid Dynamics Conference/35nd Aerospace Numerical Simulation Symposium (2017) (in Japanese).

Iioka, D., Fukuda, K., Okamoto, M., Sasaki, D., Shimoyama, K. and Obayashi, S., Computational analysis of thin airfoils under low-reynolds number flow using block-structured cartesian mesh, 55th AIAA Aerospace Science Meeting, (AIAA SciTech Forum) (2017), AIAA Paper 2017-0546.

Kroo, I. and Kunz, P., Mesoscale flight and miniature rotorcraft development, Mueller, T. J. ed., Fixed and flapping wing aerodynamics for micro air vehicle applications (2001), pp.503-517, AIAA.

Nakahashi K. and Kim, L. S., Building-cube method for large-scale, high resolution flow computations, 42nd AIAA Aerospace Sciences Meeting and Exhibit (2004), AIAA Paper 2004-434.

Okamoto, M., Yasuda K. and Azuma, A., Aerodynamic characteristics of the wings and body of a dragonfly, Journal of Experimental Biology, Vol.199 (1996), pp.281-294.

Wood, R. J., Finio, B., Karpelson, M., Ma, K., Perez-Arancibia, N. O., Sreetharan, P. S., Tanaka, H. and Whitney, J. P., Progress on pico air vehicles, The International Journal of Robotics Research, Vol.31, Issue 11 (2012), pp.12921302. 